\title{
Network Pharmacological Mechanism Research of Herba Drynariae Rhizoma-Epimedii Folium in Treating Osteoarthritis
}

\author{
Zonghui Dai ${ }^{*}$, Hui Chen ${ }^{1 *}$, Yongtao Xu ${ }^{2 \#}$ \\ ${ }^{1}$ Department of Medicine, Yangtze University, Jingzhou, China \\ ${ }^{2}$ Department of Orthopedics, Jingzhou Central Hospital, Second Clinical Medical Hospital, Yangtze University, Jingzhou, China \\ Email: "xyt7512@sohu.com
}

How to cite this paper: Dai, Z.H., Chen, H. and Xu, Y.T. (2021) Network Pharmacological Mechanism Research of Herba Drynariae Rhizoma-Epimedii Folium in Treating Osteoarthritis. Yangtze Medicine, 5, 90-105.

https://doi.org/10.4236/ym.2021.52010

Received: June 14, 2020

Accepted: April 12, 2021

Published: April 15, 2021

Copyright $\odot 2021$ by author(s) and Scientific Research Publishing Inc. This work is licensed under the Creative Commons Attribution International License (CC BY 4.0).

http://creativecommons.org/licenses/by/4.0/ (c) (i) Open Access

\begin{abstract}
Objective: To investigate the potential mechanism of Drynariae Rhizoma-Epimedii Folium in the treatment of osteoarthritis (OA) based on network pharmacology. Methods: The potential active constituents and targets of Drynariae Rhizoma-Epimedii Folium were screened through the traditional Chinese medicine (TCM) systems pharmacology database and analysis platform (TCMSP). Genecards database is used to find relevant targets of OA. The targets of "Drynariae Rhizoma-Epimedii Folium" were mapped to the targets of $\mathrm{OA}$, and used Cytoscape software to build a "drug-ingredient-target-di- sease" regulatory network and protein protein interaction (PPI) network. R software was used to analyze the Gene ontology (GO) function and Kyoto encyclopedia of genes and genomes (KEGG) pathway enrichment of traditional Chinese medicine-disease targets. Results: Thirty-four effective ingredients and 130 traditional Chinese medicine-disease targets were screened out for the treatment of OA. The GO functions of traditional Chinese medicine-disease targets mainly included cytokine activity, cytokine receptor binding, nuclear receptor activity, transcription factor activity, proximal promoter DNA-binding transcription activator activity, DNA-binding transcription activator activity, phosphatase binding and so on. KEGG pathways involved in traditional Chinese medicine-disease targets mainly included TLR4 signaling pathway, TNF signaling pathway, IL-17 signaling pathway, MAPK signaling pathway, PI3K/AKT signaling pathway, apoptotic signaling pathway and so on. Conclusion: Network pharmacology may predict the multiple targets and multiple signaling pathways in Drynariae Rhizoma-Epimedii Folium treatment for OA, providing new ideas for future research.
\end{abstract}

\footnotetext{
${ }^{\star}$ Authors contributed equally.

\#Corresponding author.
} 


\section{Keywords}

Drynariae Rhizoma, Epimedii Folium, Osteoarthritis, Network

Pharmacology, Target, Signal Pathway

\section{Introduction}

Osteoarthritis $(\mathrm{OA})$ is a common and complex chronic degenerative disease. It is mainly related to factors such as age and weight [1]. Clinically, patients with OA often present symptoms, such as injury, pain and stiffness of articular cartilage. Eventually, it leads to the inconvenience of patients' mobility and the decrease of patients' quality of life, and the social and medical burden is also increased [2] [3]. At present, the treatment of early OA is mainly based on symptomatic and drug-based palliative treatment such as alleviating pain, relieving symptoms and preventing disease progression. The guideline first chooses non-steroidal anti-inflammatory drugs, but for older patients, long-term use of non-steroidal drugs, the risk of drug-like gastrointestinal bleeding is greatly increased, and severe cases can be life-threatening. Surgical treatment of artificial joint replacement is feasible for patients with advanced bone and joint damage. However, this method is only suitable for a small number of patients, and is not suitable for older patients with cardiopulmonary insufficiency. Therefore, it is still suitable for the treatment of osteoarthritis. Therefore, there is no substantial progress in the treatment of osteoarthritis [4]. Therefore, it is of great practical significance to study the pathogenesis and treatment measures related to OA.

With a history of thousands of years, Traditional Chinese medicine (TCM) is safe, cheap, effective and suitable for the national physique. The treatment of OA with TCM has become a hot topic for many researchers [5]. As the prescription in the treatment of OA, Drynariae Rhizoma-Epimedii Folium has the functions of tonifying liver and kidney, strengthening muscles and bones [6]. Research suggests that the total flavonoids in Drynariae Rhizoma have a variety of anti-osteoarthritis mechanisms [7]; epimedii Folium has the functions of invigorating the kidney and strengthening yang, strengthening the muscles and bones etc. They are all common TCM for the prevention and treatment of OA [8]. Studies have shown that Drynaria Fortunei is often used for bone repair after trauma. Yang et al. [6] found that Gusuibu inhibits the NF- $\mathrm{BB} / \mathrm{I} \kappa \mathrm{B} \alpha / \mathrm{IKK}$ signaling pathway, and then down regulates the expression of cell adhesion molecules and chemokines. It is believed that Drynaria Fortunei may be an effective treatment for osteoarthritis. We know that osteoarthritis is a kind of articular cartilage damage. Zhou et al. [9] found that the main extract of Epimedii Folium can interfere with the chondrocyte damage induced by IL- $1 \beta$ by inhibiting cell apoptosis. Icariin is an active flavonoid component of Epimedii Folium, studies have shown that Icariin treatment promotes chondrocyte viability by promoting HIF-1 $\alpha$ expression and anaerobic glycolysis [10]. Due to the large number of 
chemical components of traditional Chinese medicine, it has been a heavy workload to use animal experiments to screen anti-osteoarthritis active ingredients and targets, and current studies have failed to fully elaborate its mechanism of action.

Network pharmacology is a new method of pharmacological data analysis, which forms an interactive network between "drug - gene - disease" to establish the interaction between multi-compound, multi-target and multi-pathway, and to clarify the pharmacological mechanism or disease pathogenesis mechanism of "multi-component, multi-target and multi-pathway" [11]. From the systems perspective and at molecular level, with the method of network pharmacology, this study studies the main effective components and potential molecular mechanism of Drynariae Rhizoma-Epimedii Folium in the treatment of OA and analyzes its key targets and signal pathways to provide theoretical basis for the follow-up mechanism of drug treatment.

\section{Materials and Methods}

\subsection{Research Ideas}

Refer Figure 1 for the experimental flow chart of the treatment of OA with Drynariae Rhizoma-Epimedii Folium based on network pharmacology.

\subsection{Screening of Active Ingredients and Target Prediction of Drynariae Rhizoma-Epimedii Folium}

Traditional Chinese Medicine Systems Pharmacology Database and Analysis Platform (TCMSP) [12] is a unique pharmacological platform of Chinese herbal medicine system. It uses the relationship between drugs and disease to provide a platform for the study of the active ingredients of Chinese herbal medicine. First, retrieve on TCMSP platform (http://tcmspw.com/tcmsp.php) to obtain all the ingredients of "Drynariae Rhizoma-Epimedii Folium"; second, set the screening condition as: drug oral bioavailability $(\mathrm{OB}) \geq 30 \%$, drug likeness (DL) $\geq 0.18$ to finally obtain the active ingredients of "Drynariae Rhizoma-Epimedii

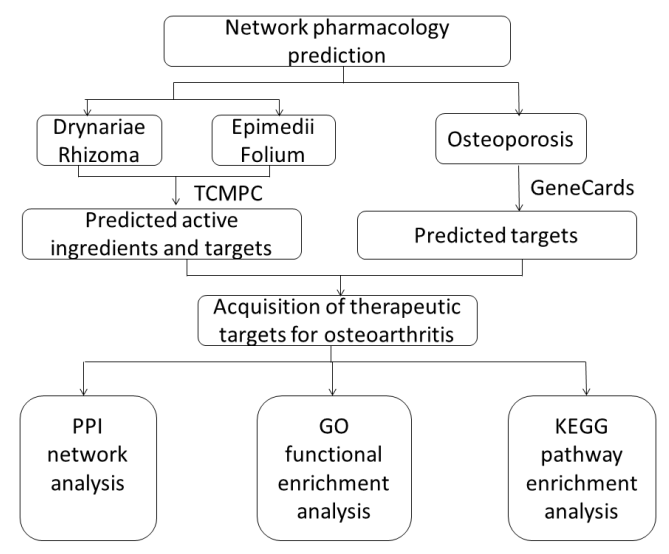

Figure 1. Network pharmacology research flow chart for Drynariae Rhizoma-Epimedii Folium in treatment of $\mathrm{OA}$. 
Folium". Based on the obtained active ingredients above, select "Related Targets" to collect the target of active ingredients of Drynariae Rhizoma-Epimedii Folium. Then, add gene names to the selected targets with online database Uniprot (https://www.uniprot.org/).

\subsection{Acquisition of Therapeutic Targets for $\mathrm{OA}$}

GeneCards (https://www.genecards.org/) is a comprehensive database of disease-related genes. It contains human gene annotation and prediction information. By inputting "osteoarthritis" into the retrieval box, the disease-related targets are obtained. With R language software (V3.5.1), the target of Drynariae Rhizoma-Epimedii Folium is mapped with OA related genes, and the Venn diagram of the common target of TCM and disease is obtained, hence, the relative targets of Drynariae Rhizoma-Epimedii Folium in the treatment of OA are obtained.

\subsection{Construct "Drug - Ingredient - Target - Disease"}

Based on the active ingredients of Drynariae Rhizoma-Epimedii Folium, the target regulated by the active ingredients, and the correlation between the target and OA, the regulatory network of "Drug - Ingredient - Target - Disease" is constructed with cytoscape (Version 3.7.1). In this network, node represents drug (Drynariae Rhizoma-Epimedii Folium), active ingredient, disease (osteoarthritis) and target. And edge represents the interaction between the drug and the active ingredient, the active ingredient and the target, and the target and OA.

\subsection{Target Protein Interaction Network of Drynariae Rhizoma-Epimedii Folium in the Treatment of $\mathrm{OA}$}

Input target of Drynariae Rhizoma-Epimedii Folium in the treatment of OA into STRING (https://string-db.org/) online database, click "Homo sapiens" to retrieve. Take the confidence score greater than 0.7 as the screening parameter, download the corresponding result data. By analyzing the data with $\mathrm{R}$ language, the degree of the target in the protein protein interaction (PPI) network is obtained, and it is presented in the form of a bar graph. Finally, import the downloaded PPI result file into the Cytoscape software for visualization. The size and color depth of the node can both represent the size of the target degree value.

\subsection{Gene Ontology (G0) Functional Enrichment Analysis and Kyoto Encyclopedia of Genes and Genomes (KEGG) Pathway Enrichment Analysis}

The obtained TCM-disease targets are calculated using R language to obtain the GO (gene ontology) functional enrichment analysis and KEGG (Kyoto encyclopedia of genes and genomes) pathway enrichment analysis. First, install the Bioconductor package on R software (http://org/biocLite.R) and set $p<0.05, q<$ 0.05 , and present the analysis results in the form of bar chart and bubble chart. 


\subsection{Statistical Analysis}

The collected drug data of Drynariae Rhizoma-Epimedii Folium use Perl language to run the relevant script file to obtain the target gene name of the drug. Use Uniprot as the drug target protein to screen, and then annotate the drug gene name to get the abbreviation of the drug gene. After removing the repeated genes, 214 drug-related genes were finally obtained. The obtained 214 drug genes and 2854 disease genes of osteoarthritis were used to draw Venny diagrams using Perl script files, and the common genes of drugs and osteoarthritis were obtained, that is, the intersection genes. Import the obtained drug-disease intersection genes into the Cytoscape 3.7.1 software for visual network construction, display the drug-active ingredient-target-disease network interaction network diagram, and import the intersection genes into String Database, and select the detection species as "Homo sapiens", select "medium confidece as 0.07 " as the screening criteria, and delete discrete or unrelated gene nodes. Finally obtain the corresponding PPI network. Finally, the key genes were imported into R language, and GO enrichment analysis and KEGG pathway analysis were performed.

\section{Results}

\subsection{Screening and Target Collection of Active Ingredients of Drynariae Rhizoma-Epimedii Folium}

Retrieve from TCMSP database to obtain the active ingredients and related targets of Drynariae Rhizoma-Epimedii Folium, select ingredients with $\mathrm{OB} \geq 30 \%$ and $\mathrm{DL} \geq 0.8$. Combined with literature reports, a total of 34 active ingredients were obtained, as shown in Table 1.

\subsection{Prediction of the Target of Drynariae Rhizoma-Epimedii Folium in the Treatment of $\mathrm{OA}$}

By taking the intersection of the OA related target and the Drynariae Rhizoma-Epimedii Folium target with $\mathrm{R}$ language software, a total of 130 targets of Drynariae Rhizoma-Epimedii Folium in the treatment of OA were obtained. There were $2854 \mathrm{OA}$ related targets, and 214 targets of active ingredients of Drynariae Rhizoma-Epimedii Folium. Refer Figure 2 for more details.

\subsection{Construct "Drug - Ingredient - Target - Disease" Network and Analyze}

The corresponding relationship between Drynariae Rhizoma-Epimedii Folium and its corresponding active ingredients, active ingredients and target genes, and target genes and osteoarthritis and its properties were imported into Cytoscape software to construct "Drug - Ingredient - Target - Disease" regulatory network. Refer Figure 3 for more details. In the network, the blue quadrilateral represents the drug "Drynariae Rhizoma", "Epimedii Folium", the green inverted triangle represents the active ingredients, the red hexagon represents osteoarthritis, and 
Table 1. Basic information of the main active ingredients of Drynariae Rhizoma-Epimedii Folium.

\begin{tabular}{|c|c|c|c|c|}
\hline MOL ID & Molecule name & $\begin{array}{l}\text { OB } \\
(\%)\end{array}$ & DL & $\begin{array}{l}\text { Number } \\
\text { of targets }\end{array}$ \\
\hline MOL000098 & quercetin & 46.43 & 0.28 & 136 \\
\hline MOL000422 & kaempferol & 41.88 & 0.24 & 114 \\
\hline MOL000006 & luteolin & 36.16 & 0.25 & 107 \\
\hline MOL000358 & beta-sitosterol & 36.91 & 0.75 & 38 \\
\hline MOL004328 & naringenin & 59.29 & 0.21 & 37 \\
\hline MOL000449 & Stigmasterol & 43.83 & 0.76 & 31 \\
\hline MOL004380 & $\begin{array}{l}\text { C-Homoerythrinan, } \\
\text { 1,6-didehydro-3,15,16-trimethoxy-, (3.beta.)- }\end{array}$ & 39.14 & 0.49 & 30 \\
\hline MOL004373 & Anhydroicaritin & 45.41 & 0.44 & 26 \\
\hline MOL004391 & 8-(3-methylbut-2-enyl)-2-phenyl-chromone & 48.54 & 0.25 & 23 \\
\hline MOL003542 & 8-Isopentenyl-kaempferol & 38.04 & 0.39 & 18 \\
\hline MOL001978 & Aureusidin & 53.42 & 0.24 & 17 \\
\hline MOL009091 & xanthogalenol & 41.08 & 0.32 & 13 \\
\hline MOL003044 & Chryseriol & 35.85 & 0.27 & 12 \\
\hline MOL000492 & $(+)$-catechin & 54.83 & 0.24 & 11 \\
\hline MOL001040 & $\begin{array}{c}\text { (2R)-5,7-dihydroxy-2-(4-hydroxyphenyl) } \\
\text { chroman-4-one }\end{array}$ & 42.36 & 0.21 & 9 \\
\hline MOL005190 & eriodictyol & 71.79 & 0.24 & 9 \\
\hline MOL001792 & DFV & 32.76 & 0.18 & 8 \\
\hline MOL002914 & Eriodyctiol (flavanone) & 41.35 & 0.24 & 8 \\
\hline MOL004384 & Yinyanghuo C & 45.67 & 0.5 & 7 \\
\hline MOL004396 & $\begin{array}{l}\text { 1,2-bis(4-hydroxy-3-methoxyphenyl) } \\
\text { propan-1,3-diol }\end{array}$ & 52.31 & 0.22 & 7 \\
\hline MOL004386 & Yinyanghuo E & 51.63 & 0.55 & 6 \\
\hline MOL009078 & davallioside A_qt & 62.65 & 0.51 & 6 \\
\hline MOL004382 & Yinyanghuo A & 56.96 & 0.77 & 5 \\
\hline MOL001645 & Linoleyl acetate & 42.1 & 0.2 & 4 \\
\hline MOL000359 & sitosterol & 36.91 & 0.75 & 3 \\
\hline MOL000569 & digallate & 61.85 & 0.26 & 3 \\
\hline MOL001510 & 24-epicampesterol & 37.58 & 0.71 & 2 \\
\hline MOL001771 & poriferast-5-en-3beta-ol & 36.91 & 0.75 & 2 \\
\hline MOL004367 & olivil & 62.23 & 0.41 & 2 \\
\hline MOL004388 & $\begin{array}{l}\text { 6-hydroxy-11,12-dimethoxy-2,2- } \\
\text { dimethyl-1,8-dioxo-2,3,4,8-tetrahydro-1H- } \\
\text { isochromeno[3,4-h] isoquinolin-2-ium }\end{array}$ & 60.64 & 0.66 & 2 \\
\hline MOL004427 & Icariside A7 & 31.91 & 0.86 & 2 \\
\hline MOL000622 & Magnograndiolide & 63.71 & 0.19 & 2 \\
\hline MOL009061 & 22-Stigmasten-3-one & 39.25 & 0.76 & 1 \\
\hline MOL009075 & cycloartenone & 40.57 & 0.79 & 1 \\
\hline
\end{tabular}




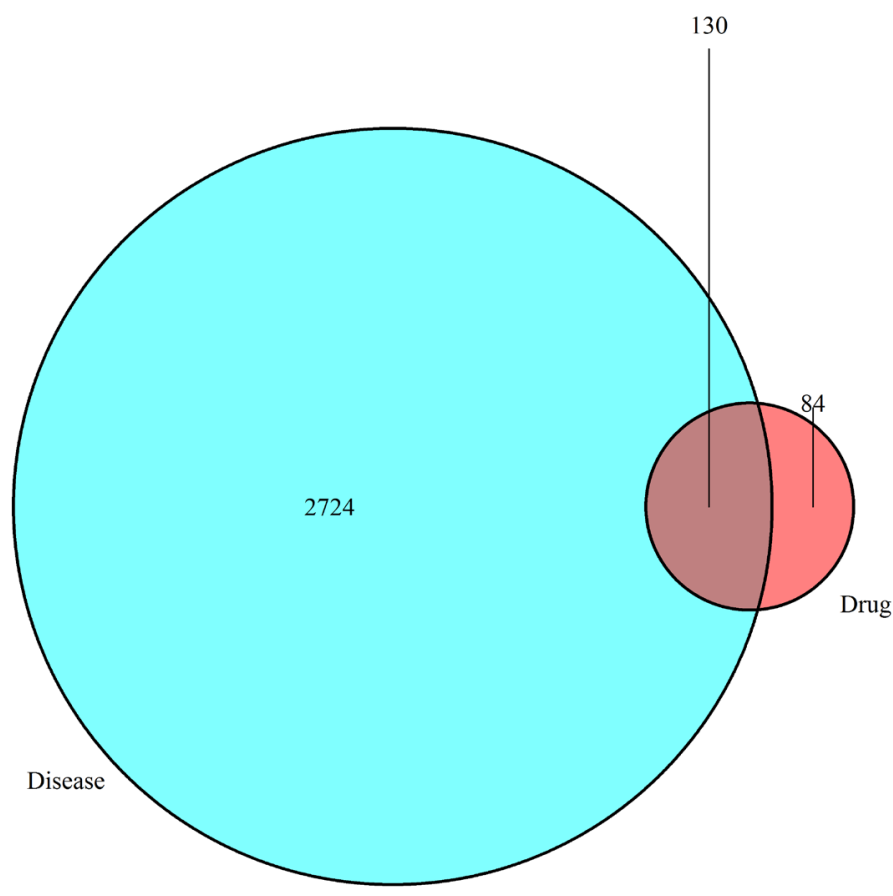

Figure 2. Venn diagram of common target for Drynariae Rhizoma-Epimedii Folium and OA-related targets.

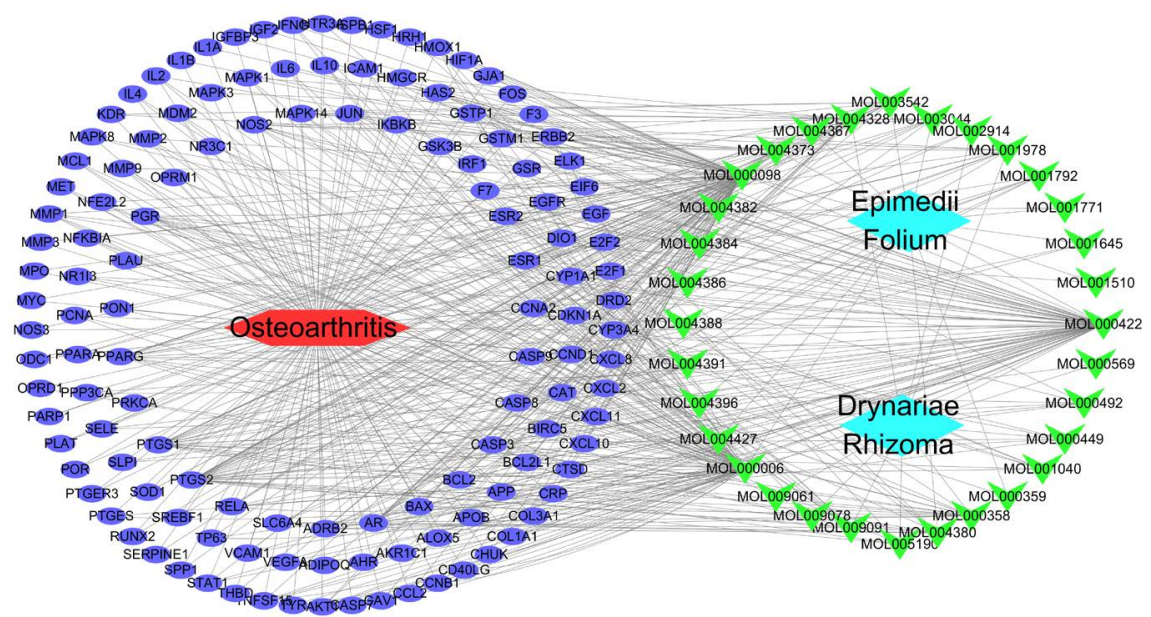

Figure 3. "Drug-ingredient-target-disease" network of Drynariae Rhizoma-Epimedii Folium for OA.

the purple oval represents the target. When a node connects more points in the network, it indicates that this node has a higher degree value, and it may play a key role in the network. The network consists of 165 nodes, including 32 nodes representing the active ingredients of Drynariae Rhizoma-Epimedii Folium (compound Magnograndiolide (MOL000622), Cycloartenone (MOL009075) have no relevant targets), and 130 nodes representing the target and 568 edges. From the regulatory network diagram, it can be seen that multiple active ingredients can correspond to one target, or one active ingredient corresponds to multiple targets. This indicates that Drynariae Rhizoma-Epimedii Folium can 
play a pharmacological role through the synergy of multiple ingredients and multiple targets. The top 3 compounds according to the ranking of degree value are: quercetin (degree value: 95), luteolin (degree value: 82) and kaempferol (degree value: 68 ).

\subsection{Construct and Analyze PPI network of Key Targets}

From Figure 4, it shows that PPI network consists of 130 nodes and 1054 edges. The darker the color and the larger the shape of the node represent the greater the degree value of the node. The higher the degree value of the target in the network, the more likely it is to become the core target in the PPI network. The degree values of the targets in the network are ranked as AKT1, IL6, JUN, MAPK8, MAPK1, VEGFA, etc., please refer Figure 5 for more details. The anti OA mechanism of Drynariae Rhizoma-Epimedii Folium may be related to targets of AKT1, IL6, JUN, MAPK8, MAPK1, VEGFA etc.

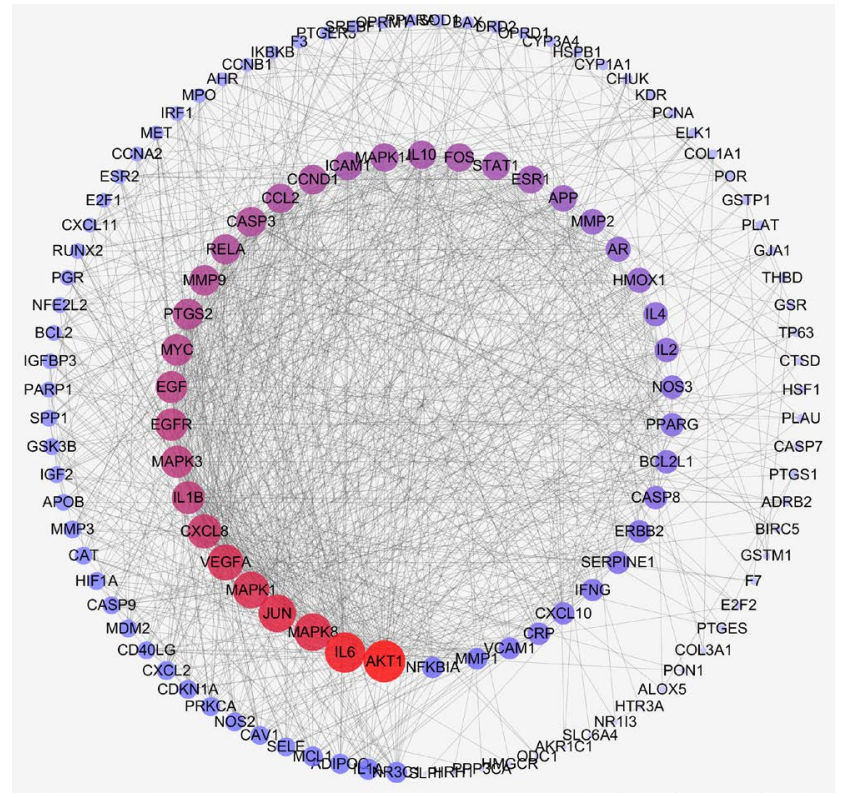

Figure 4. PPI network of Drynariae Rhizoma-Epimedii Folium and OA targets.

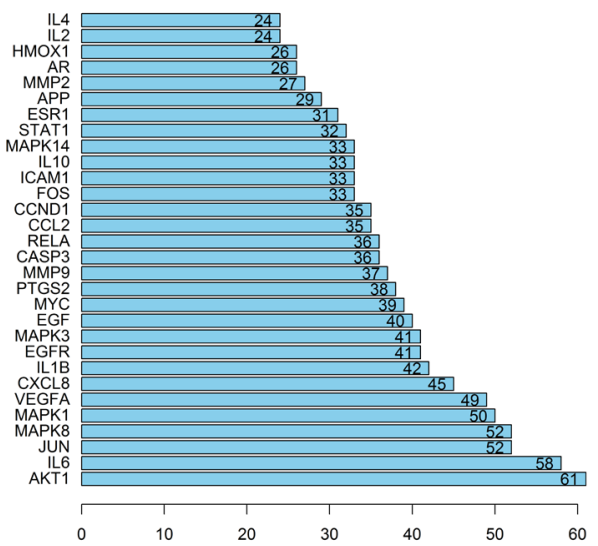

Figure 5. Targets with a higher median in PPI networks. 


\subsection{GO Functional Enrichment Analysis}

The GO functional enrichment analysis for the key targets of Drynariae Rhizoma-Epimedii Folium in the treatment of OA is shown in Figure 6. The GO function mainly involves cytokine activity, cytokine receptor binding, nuclear receptor activity, transcription factor activity, proximal promoter DNA binding transcription activator activity, DNA binding transcription activator activity, phosphatase binding, etc.

\subsection{KEGG Pathway Enrichment Analysis}

The KEGG pathway enrichment analysis for the key targets of Drynariae Rhizoma-Epimedii Folium in the treatment of OA is shown in Figure 7. KEGG pathway mainly involves: Kaposi's sarcoma associated herpesvirus infection signaling pathway, human cytomegalovirus infection signaling pathway, AGE-RAGE signaling pathway in diabetic complications, Hepatitis B signaling pathway, fluid shear stress and atherosclerosis, TNF signaling pathway, IL-17 signaling pathway, MAPK signaling pathway, prostate cancer signaling pathway, EB virus infection signal pathway, apoptosis of cells etc.

\section{Discussions}

As a relatively common joint disease, $\mathrm{OA}$ often causes middle-aged and elderly people to lose their ability to exercise, and brings great inconvenience to patients. According to the study, the incidence of $\mathrm{OA}$ in the global population aged

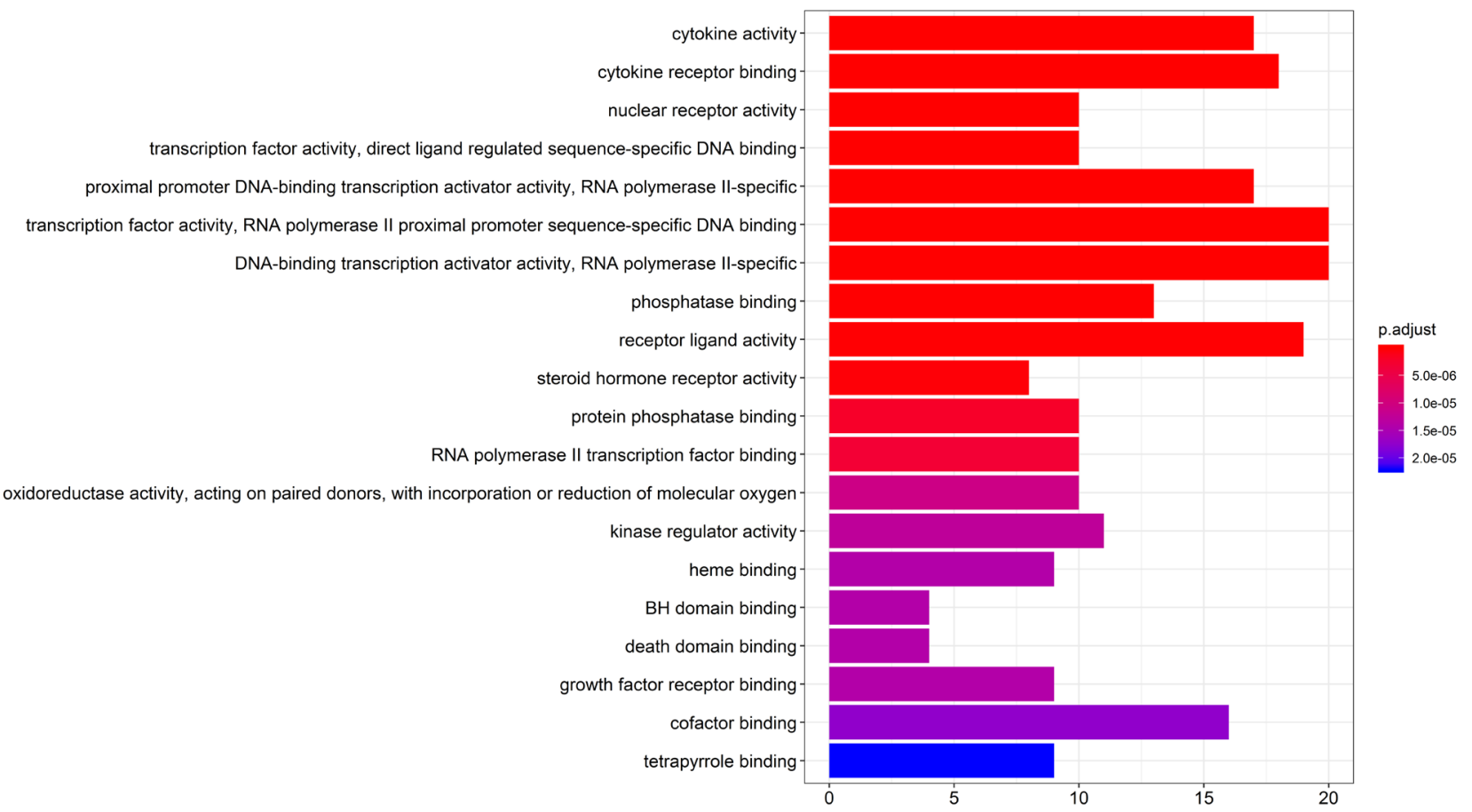

Figure 6. Barplot for GO functional enrichment analysis. The letters on the left represent GO entries; the numbers represent the number of genes enriched on GO entries; the bar graph represents the genes enriched on GO; the $P$ value represents the significance of enrichment, the redder the color, the higher the degree of enrichment, the smaller the corresponding $P$ value. 


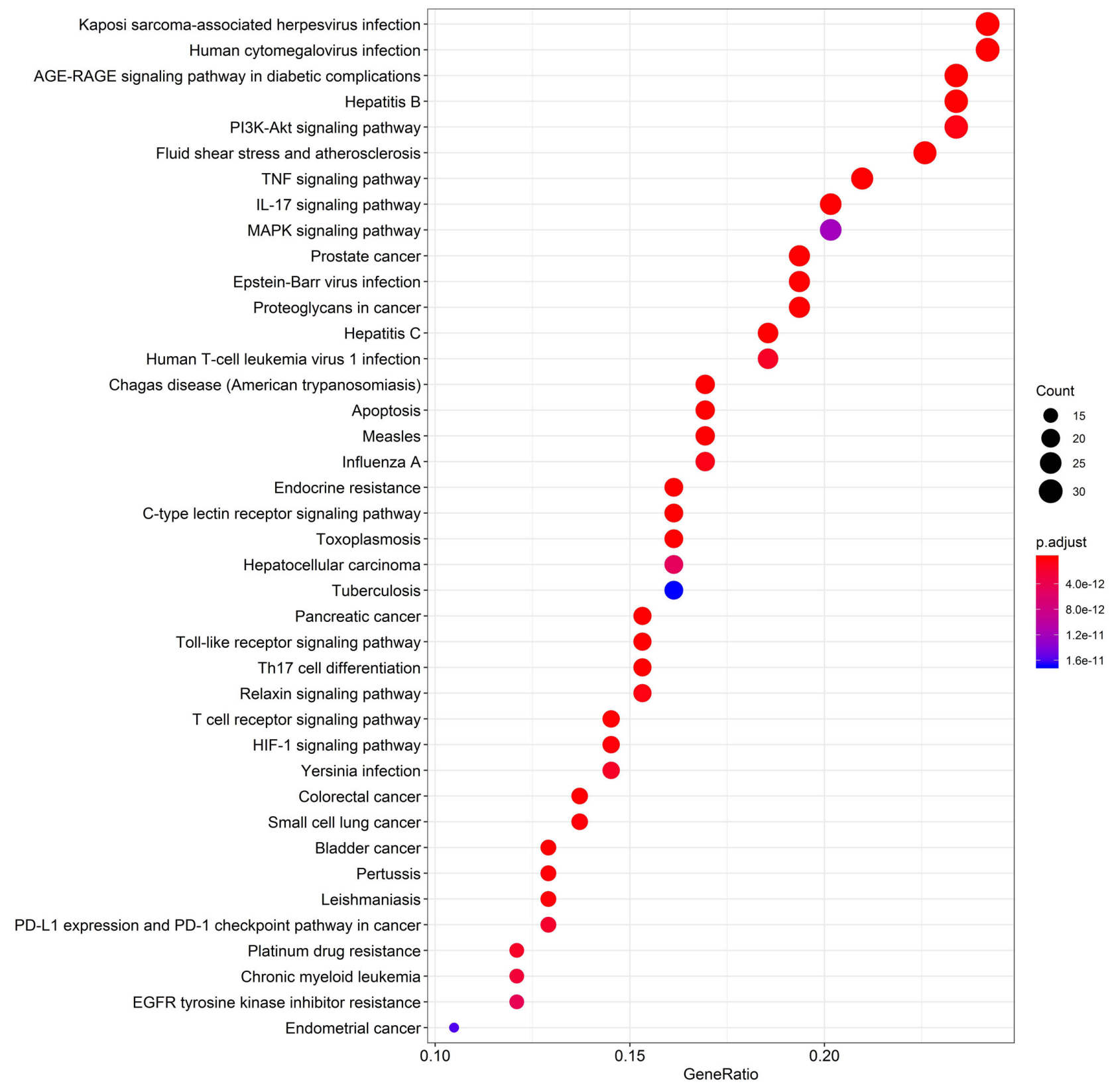

Figure 7. Dotplot for KEGG pathway enrichment analysis. The letters on the left represent the name of the KEGG signaling pathway; the numbers represent the proportion of genes; the size of the circle represents the number of enriched genes; the color represents the $P$ value.

over 65 years is as high as $50 \%$ [13]. In traditional Chinese medicine, OA belongs to the scope of "Bi disease," middle aged and elderly people tend to have incoordination between the liver and the spleen, and deficiency of kidney essence, resulting in the body imbalance of Yin and Yang, deficiency of Qi and blood, loss of nourishment of bones, and eventually have arthralgia syndrome [5]. Due to the convenience and safety of application and the small side effects, TCM in the treatment of OA has achieved remarkable efficacy. In the early treatment of patients, it can improve the efficacy, slow down the development of 
the disease, as well as significantly improving the quality of life of patients.

Drynariae Rhizoma is the dry rhizome of Dryopteris crassipes. With the functions of strengthening bone, tonifying kidney, relieving pain, regulating lipid and anti-inflammation, it has a certain effect on the treatment of OA [7]. Epimedii Folium has the functions of tonifying kidney-yang, strengthening muscles and bones, dispelling wind-damp, and is extensively applied in the clinical treatment of OA, osteoporosis and other diseases [14] [15]. At present, TCM has good clinical efficacy in the treatment of OA, but its molecular mechanism in the treatment of OA remains unclear. Therefore, from the perspective of multi-ingredient, multi-target and multi-pathway of Drynariae Rhizoma-Epimedii Folium, this paper clarifies its mechanism with the method of network pharmacology.

Based on this study, Drynariae Rhizoma-Epimedii Folium has the following chemical components with anti OA: quercetin, kaempferol and luteolin. The quercetin has many functions such as antioxidant stress, anti-inflammatory, inhibition of osteoclast activation, etc. Some studies have shown that quercetin mainly slows the development of chronic diseases such as OA, rheumatoid arthritis, osteoporosis etc. by reducing the release of pro-inflammatory factors, reducing oxidative stress, and inhibiting the degradation of extracellular matrix [16]. According to relevant experimental studies, quercetin can play a better role in the treatment of OA by down-regulating the expression of TNF- $\alpha$, IL-1 $\beta$, IL-6 and other inflammatory factors [17]. In the rat model of OA, Qiu et al. [18] found that quercetin could down-regulate the release of MMP-13 mediated by IL- $1 \beta$, so as to improve the degeneration of OA by inhibiting the degradation of cartilage extracellular matrix. Kaempferol has the function of inhibiting adipogenesis, inflammation, osteoblast apoptosis etc. It can also protect bones by regulating the semaphore receptor, bone morphology protein 2 (BMP-2), nuclear factor- $\kappa B(N F-\kappa B)$, and mitogen activated protein kinase (MAPK) and other signaling pathways [19]. Other studies have found that by down regulating the expression of JNK, p38 and MAPK genes, luteolin can inhibit the proliferation of chondrocytes. At the same time, it can also down regulating the release of inflammatory factors such as NO, TNF- $\alpha$ and IL-6, so as to reduce inflammation and protect articular chondrocytes [20]. Therefore, it can be seen that the active ingredients in Drynariae Rhizoma-Epimedii Folium are diverse in protecting OA and play a major role in terms of anti-inflammatory, oxidative stress, apoptosis.

From PPI network analysis, the core targets obtained mainly include AKT1, IL6, JUN, MAPK8, MAPK1, VEGFA, etc. These core target genes may participate in the development of OA through various biological processes such as cytokine activity, cytokine receptor binding, nuclear receptor activity, transcription factor activity, proximal promoter DNA binding transcriptional activator activity, DNA binding transcription activator activity, phosphatase binding etc. for anti OA. AKT1 is the protein kinase encoding $\alpha$ serine/threonine. It can be activated by extracellular signal depending on PI3K. In OA, chondrocytes can 
inhibit bone growth, cartilage calcification, and osteophyte formation through the PI3K/AKT signaling pathway [21]. Knocking out the AKT1 gene delays bone formation [22]. IL-6 is a significant inflammatory molecule for inflammation and immune responses. Relevant research reports show that IL6 is associated with pain in hip OA, and is highly expressed in the patient's chondrocytes and synovial fluid [23]. JUN is also called as transcription factor AP-1, which is a dimer composed of c-jun protein and $\mathrm{c}$-fos protein family members. With the function of regulating the release of many inflammatory factors, such as IL-1 $\beta$, TNF- $\alpha$, IL-6, IL-8, etc., activated AP-1 is called the third messenger of intracellular signaling, and it also plays a regulatory role in cell proliferation, differentiation, apoptosis and inflammation [24]. Lim et al. [25] study found that the blocking of the signaling pathways p38 MAPK/AP-1 and JAK2/STAT1/2 can significantly reduce the release of MMP-13 in chondrocytes treated by IL- $1 \beta$, thus exerting the effect of protecting cartilage degeneration [26]. Vascular endothelial growth factor (VEGF) family has been shown to play a crucial role both in the control of catabolism of articular cartilage and angiogenesis. This is a key step in the endochondral ossification of mesenchymal progenitor cells [27].

The KEGG signaling pathway enrichment results show that Drynariae Rhizoma-Epimedii Folium can play its role through multiple signal pathways, mainly including TLR4 signaling pathway, TNF signaling pathway, IL-17 signaling pathway, MAPK signaling pathway, PI3K/AKT signaling pathway and apoptosis signaling pathway, etc. TLR4 signaling pathway, TNF signaling pathway, IL-17 signaling pathway, etc. are closely related to inflammation. Both IL-1 AND TNF-aare key inflammatory factors in the pathogenesis of OA. TLR4 signaling pathway activates downstream NF- $\mathrm{kB}$, regulates the release of pro-inflammatory factors such as IL- $1 \beta$, IL-6, IL-17, TNF- $\alpha$, and produces an inflammatory cascade reaction. Many studies found that by inhibiting NF- $\mathrm{kB}$ and blocking the inflammatory signal pathway, the destruction of cartilage in OA can be significantly reduced [28]. MAPKs signaling pathway is one of the most important signaling pathways in regulating OA. It may be involved in the growth, differentiation and apoptosis of chondrocytes, and it may also induce the secretion of MMPs, which can lead to the degradation of cartilage matrix [29]. MAPKs signaling pathway can also regulate the differentiation and maturation of osteoblasts to improve bone density and calcification; by linking with other signaling pathways such as downstream ERK, p38, JNK, it regulates the production of inflammatory factors, promotes cartilage dexterization, osteophyte formation and extracellular matrix degradation, which plays a role in the pathogenesis of OA [30]. PI3K-AKT signaling pathway is a common regulation of apoptosis-related signaling pathway. Some studies demonstrates that by activating the PI3K-AKT signaling pathway, the expression of Caspase- 3 and Caspase- 8 can be increased, and the anti-apoptosis protein Bcl-2 is reduced to induce chondrocyte apoptosis [31]. Articular chondrocyte apoptosis is the biological basis of OA degeneration. The unbalanced development between excessive apoptosis of cartilage cells and osteoclast proliferation leads to bone reconstruc- 
tion [32] [33]. With collaboration between apoptosis signaling pathway and upstream signaling pathway of MAPKs, PI3K-AKT and NF-kB and downstream signaling pathway of exogenous death receptor and endogenous mitochondrial pathway, so the apoptosis signal is released to regulate apoptosis of chondrocytes [34].

In this study, we used data mining and network pharmacology to explain the possible mechanism of Drynariae Rhizoma and Epimedii Folium in the treatment of osteoarthritis. Moreover, our results give a scientific basis to further explore the mechanism of Drynariae Rhizoma and Epimedii Folium in the treatment of osteoarthritis, and reduce unnecessary time and economic cost. However, the relevant pathological mechanism needs to be further verified in experiments.

\section{Conclusion}

In conclusion, this paper studied the molecular mechanism of Drynariae Rhizoma-Epimedii Folium protecting OA based on network pharmacology. The mechanism may function mainly through targets of AKT1, IL6, JUN, MAPK8, MAPK1, VEGFA, etc. and pathways of TLR4, TNF, IL-17, MAPK, PI3K/AKT, apoptosis, etc. This study has laid the foundations for later study on the mechanism of the treatment of OA with Drynariae Rhizoma-Epimedii Folium, and provided a new idea and direction for the R \& D of TCM and clinical medication guidance.

\section{Acknowledgements}

This work was supported by Joint Foundation of the Health Commission of Hubei Province (WJ2016Y13).

\section{Conflicts of Interest}

The authors declare no conflicts of interest regarding the publication of this paper.

\section{References}

[1] Wei, Y., Zheng, D., Guo, X., et al. (2018) Transient Receptor Potential Channel, Vanilloid 5, Induces Chondrocyte Apoptosis in a Rat Osteoarthritis Model through the Mediation of $\mathrm{Ca}^{2+}$ Influx. Cellular Physiology and Biochemistry, 46, 687-698. https://doi.org/10.1159/000488725

[2] Maudens, P., Jordan, O. and Allemann, E. (2018) Recent Advances in Intra-Articular Drug Delivery Systems for Osteoarthritis Therapy. Drug Discovery Today, 23, 1761-1775. https://doi.org/10.1016/j.drudis.2018.05.023

[3] Hiligsmann, M., Cooper, C., Arden, N., et al. (2013) Health Economics in the Field of Osteoarthritis: An Expert's Consensus Paper from the European Society for Clinical and Economic Aspects of Osteoporosis and Osteoarthritis (ESCEO). Seminars in Arthritis and Rheumatism, 43, 303-313.

https://doi.org/10.1016/j.semarthrit.2013.07.003 
[4] Lambova, S.N. and Muller-Ladner, U. (2018) Osteoarthritis-Current Insights in Pathogenesis, Diagnosis and Treatment. Current Rheumatology Reviews, 14, 91-97. https://doi.org/10.2174/157339711402180706144757

[5] Tsai, P.W., Lee, Y.H., Chen, L.G., Lee, C.J. and Wang, C.C. (2018) In Vitro and in Vivo Anti-Osteoarthritis Effects of 2,3,5,4'-Tetrahydroxystilbene-2-O- $\beta$-d-Glucoside from Polygonum Multiflorum. Molecules (Basel, Switzerland), 23, 571. https://doi.org/10.3390/molecules23030571

[6] Yang, R.C., Chang, C.C., Sheen, J.M., et al. (2014) Davallia Bilabiata Inhibits TNF- $\alpha$-Induced Adhesion Molecules and Chemokines by Suppressing IKK/NFKappa B Pathway in Vascular Endothelial Cells. The American Journal of Chinese Medicine, 42, 1411-1429. https://doi.org/10.1142/S0192415X1450089X

[7] Yao, W., Zhang, H., Jiang, X., et al. (2018) Rhizoma Drynariae Effect of Total Flavonoids of on Tibial Dyschondroplasia by Regulating BMP-2 and Runx2 Expression in Chickens. Frontiers in Pharmacology, 9, 1251. https://doi.org/10.3389/fphar.2018.01251

[8] Chen, D., Su, X., Wang, N., et al. (2017) Chemical Isotope Labeling LC-MS for Monitoring Disease Progression and Treatment in Animal Models: Plasma Metabolomics Study of Osteoarthritis Rat Model. Scientific Reports, 7, Article No. 40543. https://doi.org/10.1038/srep40543

[9] Zhou, A.Z., Wang, L. and Cheng, B. (2020) Ethanol Extraction Technology of Epimedii Folium and Protective Effect of Ethanol Extract on Chondrocyte. China Journal of Chinese Materia Medica, 45, 1097-1104.

[10] Wang, P., Xiong, X., Zhang, J., et al. (2020) Icariin Increases Chondrocyte Vitality by Promoting Hypoxia-Inducible Factor-1 $\alpha$ Expression and Anaerobic Glycolysis. Knee, 27, 18-25. https://doi.org/10.1016/j.knee.2019.09.012

[11] Yang, Y.X., Ping, Z., Yingying, W., et al. (2018) Hepatoprotective Effect of San-Cao Granule on Con A-Induced Liver Injury in Mice and Mechanisms of Action Exploration. Frontiers in Pharmacology, 9, 624. https://doi.org/10.3389/fphar.2018.00624

[12] Ru, J., Li, P., Wang, J., et al. (2014) TCMSP: A Database of Systems Pharmacology for Drug Discovery from Herbal Medicines. Journal of Cheminformatics, 6, 13. https://doi.org/10.1186/1758-2946-6-13

[13] Li, M.H., Xiao, R., Li, J.B. and Zhu, Q. (2017) Regenerative Approaches for Cartilage Repair in the Treatment of Osteoarthritis. Osteoarthritis Cartilage, 25, 1577-1587. https://doi.org/10.1016/j.joca.2017.07.004

[14] Zhang, W., Li, R., Wang, S., Mu, F. and Jia, P. (2013) Effect of Chinese Traditional Herb Epimedium grandiflorum C. Morren and Its Extract Icariin on Osteoarthritis via Suppressing NF-kappaB Pathway. Indian Journal of Experimental Biology, 51, 313-321.

[15] Zu, Y., Mu, Y., Li, Q., Zhang, S.T. and Yan, H.J. (2019) Icariin Alleviates Osteoarthritis by Inhibiting NLRP3-Mediated Pyroptosis. Journal of Orthopaedic Surgery and Research, 14, 307. https://doi.org/10.1186/s13018-019-1307-6

[16] Hu, Y., Gui, Z., Zhou, Y., Xia, L., Lin, K., Xu, Y., et al. (2019) Quercetin Alleviates Rat Osteoarthritis by Inhibiting Inflammation and Apoptosis of Chondrocytes, Modulating Synovial Macrophages Polarization to M2 Macrophages. Free Radical Biology \& Medicine, 145, 146-160. https://doi.org/10.1016/j.freeradbiomed.2019.09.024

[17] Leyva-López, N., Gutierrez-Grijalva, E.P., Ambriz-Perez, D.L. and Heredia, J.B. (2016) Flavonoids as Cytokine Modulators: A Possible Therapy for Inflamma- 
tion-Related Diseases. International Journal of Molecular Sciences, 17, 921. https://doi.org/10.3390/ijms17060921

[18] Qiu, L., Luo, Y. and Chen, X. (2018) Quercetin Attenuates Mitochondrial Dysfunction and Biogenesis via Upregulated AMPK/SIRT1 Signaling Pathway in OA Rats. Biomedicine \& Pharmacotherapy, 103, 1585-1591.

https://doi.org/10.1016/j.biopha.2018.05.003

[19] Wong, S.K., Chin, K.Y. and Ima-Nirwana, S. (2019) The Osteoprotective Effects of Kaempferol: The Evidence from in Vivo and in Vitro Studies. Drug Design, Development and Therapy, 13, 3497-3514. https://doi.org/10.2147/DDDT.S227738

[20] Xue, J., Ye, J., Xia, Z. and Cheng, B. (2019) Effect of Luteolin on Apoptosis, MAPK and JNK Signaling Pathways in Guinea Pig Chondrocyte with Osteoarthritis. Cellular and Molecular Biology (Noisy-le-Grand, France), 65, 91-95.

https://doi.org/10.14715/cmb/2019.65.6.15

[21] Fukai, A., Kawamura, N., Saito, T., et al. (2010) Akt1 in Murine Chondrocytes Controls Cartilage Calcification during Endochondral Ossification under Physiologic and Pathologic Conditions. Arthritis and Rheumatism, 62, 826-836.

https://doi.org/10.1002/art.27296

[22] Xi, J.C., Zang, H.Y., Guo, L.X., et al. (2015) The PI3K/AKT Cell Signaling Pathway Is Involved in Regulation of Osteoporosis. Journal of Receptor and Signal Transduction Research, 35, 640-645. https://doi.org/10.3109/10799893.2015.1041647

[23] Ren, G., Lutz, I., Railton, P., et al. (2018) Serum and Synovial Fluid Cytokine Profiling in Hip Osteoarthritis: Distinct from Knee Osteoarthritis and Correlated with Pain. BMC Musculoskeletal Disorders, 19, 39. https://doi.org/10.1186/s12891-018-1955-4

[24] Fu, C.Y., Chen, M.C., Tseng, Y.S., et al. (2019) Fisetin Activates Hippo Pathway and JNK/ERK/AP-1 Signaling to Inhibit Proliferation and Induce Apoptosis of Human Osteosarcoma Cells via ZAK Overexpression. Environmental Toxicology, 34, 902-911. https://doi.org/10.1002/tox.22761

[25] Lim, H. and Kim, H.P. (2011) Matrix Metalloproteinase-13 Expression in IL- $1 \beta$-Treated Chondrocytes by Activation of the p38 MAPK/c-Fos/AP-1 and JAK/STAT Pathways. Archives of Pharmacal Research, 34, 109-117. https://doi.org/10.1007/s12272-011-0113-4

[26] Ma, D., Li, Y., Xiao, W., et al. (2020) Achyranthes Bidentata Extract Protects Chondrocytes Functions through Suppressing Glycolysis and Apoptosis via MAPK/AKT Signaling Axis. American Journal of Translational Research, 12, 142-152.

[27] Vadalà, G., Russo, F., Musumeci, M., et al. (2018) Targeting VEGF-A in Cartilage Repair and Regeneration: State of the Art and Perspectives. Journal of Biological Regulators and Homeostatic Agents, 32, 217-224.

[28] Kawai, T. and Akira, S. (2010) The Role of Pattern-Recognition Receptors in Innate Immunity: Update on Toll-Like Receptors. Nature Immunology, 11, 373-384. https://doi.org/10.1038/ni.1863

[29] Sun, H.Y., Hu, K.Z. and Yin, Z.S. (2017) Inhibition of the p38-MAPK Signaling Pathway Suppresses the Apoptosis and Expression of Proinflammatory Cytokines in Human Osteoarthritis Chondrocytes. Cytokine, 90, 135-143. https://doi.org/10.1016/j.cyto.2016.11.002

[30] Roux, P.P. and Blenis, J. (2004) ERK and p38 MAPK-Activated Protein Kinases: A Family of Protein Kinases with Diverse Biological Functions. Microbiology and Molecular Biology Reviews, 68, 320-344. 
https://doi.org/10.1128/MMBR.68.2.320-344.2004

[31] Zhang, Q., Lai, S., Hou, X., et al. (2018) Protective Effects of PI3K/Akt Signal Pathway Induced Cell Autophagy in Rat Knee Joint Cartilage Injury. American Journal of Translational Research, 10, 762-770.

[32] Kim, J.H., Jin, H.M., Kim, K., et al. (2009) The Mechanism of Osteoclast Differentiation Induced by IL-1. The Journal of Immunology, 183, 1862-1870.

https://doi.org/10.4049/jimmunol.0803007

[33] Palmieri, B., Lodi, D. and Capone, S. (2010) Osteoarthritis and Degenerative Joint Disease: Local Treatment Options Update. Acta Biomedica, 81, 94-100.

[34] Kim, H.A. and Blanco, F.J. (2007) Cell Death and Apoptosis in Osteoarthritic Cartilage. Current Drug Targets, 8, 333-345.

https://doi.org/10.2174/138945007779940025 